\title{
TOWARDS AN UNDERSTANDING OF OPERATOR FOCUS USING EYE-TRACKING IN SAFETY-CRITICAL MARITIME SETTINGS
}

\author{
Dr FB Bjørneseth, Rolls-Royce Marine AS, Norway \\ L Clarke and Dr MD Dunlop, University of Strathclyde, Scotland, UK \\ Dr S Komandur, Aalesund University College, Maritime Human Factors Laboratory, Norway
}

\section{SUMMARY}

In this paper we report on our investigations into determining foci of attention for operators during safety-critical maritime operations, in this case a dynamic positioning (DP) operation. We monitored operators carrying out operations in ship simulators while wearing eye-tracking equipment. The eye-tracking was carried out during a standard operation with normal sea conditions (2-3 m wave height and no significant wind force). For the next iteration of experiments that will be reported in our next publication, it will then be possible to test the correlation between standard operations and operations with environmental effects introduced. These investigations led to an understanding of where operators focus, for how long and estimated their pupil dilation during these operations to investigate if it can correlate with critical parts of the operation. We also investigated the difference between trainee operators and operators with considerable experience. Results are presented along with a discussion of lessons for the design and placement of computer displays and equipment for safe operations in ship bridge environments.

\section{INTRODUCTION}

Maritime operations carried out for the oil industry are safety critical . Operators must monitor multiple displays that give feedback on aspects such as accurate vessel location when operating close to offshore installations, engine status and status of loading and pumping equipment. Meanwhile they must maintain "constant" awareness of the operating deck of the vessels. During these operations deckhands are often working on deck near dangerous equipment which is being controlled remotely from the bridge. The safety issues are of greatest concern when large equipment is being used such as powerful winches during anchor handling operations and when loading/unloading during platform supply operations. The increasing use of multiple computerised systems for different aspects of monitoring and control, often with differing interfaces, introduces the risk that operators may focus too frequently and for too long on visual displays for the safety of deckhands.

With this knowledge, we are investigating both the extent of the problem and if there are recognizable visual patterns during operation that give pointers on how to better design the bridge environment to support the operator both during standard work procedures and when reaching the critical point of operation. We believe that this will further reduce the human error rate. One of the key elements of a bridge concept is to maintain a good view to the outside environment, as the operators spend the majority of their time looking at the outside scenery. It is therefore important to have no significant occlusions in the field of vision (FOV), which is vital to ensure a safe working environment. However, a competing design principle is to place displays near the normal line of sight in order to reduce the time to refocus when changing between outside and display focus.

Rolls-Royce Marine AS recently launched their new Unified Bridge which includes a complete re-design of bridge consoles, levers and maritime software. The
Unified Bridge has, as one of the first, been designed on a basis of human factors, usability and user experience research. The experiment presented in this paper is a part of a chain of connected experiments using an experimental design framework [1] that has contributed to the design of the new Rolls-Royce Unified Bridge (Figure 1).

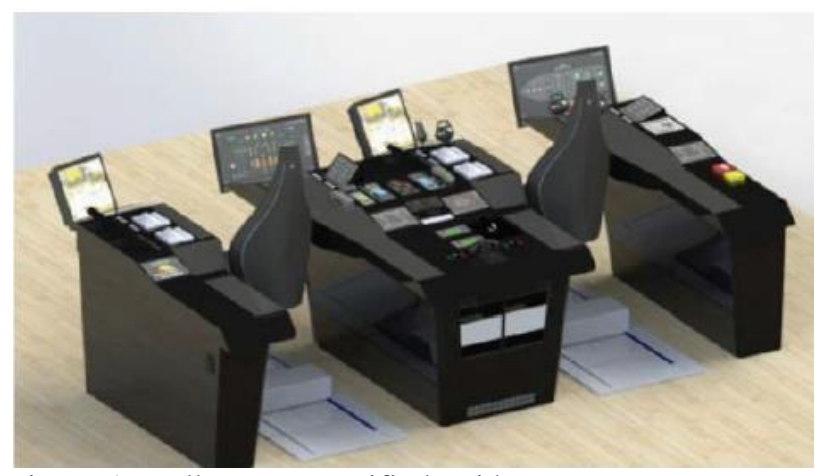

Figure 1: Rolls-Royce Unified Bridge

The framework aims at evaluating the cognitive workload of operators onboard offshore vessels (DP vessels in particular), to identify focal points of attention during operation and in the end evaluate and compare maritime graphical user interfaces situated on board utilizing previous and new design.

The experiments are informed by our understanding of the operators' work, which is in a dynamic and visually challenging environment. This understanding comes from direct observation on vessels and in working closely with operators and their trainers. This working environment demands operator attention regularly during which they must monitor and process considerable information and make decisions under conditions where task load varies across a range of their capabilities.

A Dynamic Positioning (DP) system can be defined as: $A$ computer controlled system to automatically maintain a 
ship's position and heading by using her own propellers and thrusters [2].

Its technology has developed from the first simple systems in the 1960's to today's advanced systems covering single, double and triple redundancy according to the operation's safety critical level. These vessels are used widely for delivery and maintenance operations on oil platforms and for other maritime operations, such as cable laying.

\section{MOTIVATION}

According to IMCA [3] it is clear that operator error is the highest contributor of major loss of positions of DP incidents [3]. Nocera et al. [4] says:

"Human factors and ergonomics (HF/E) research continues to demonstrate that extreme levels of mental workload decrease an individual's ability to react to incoming information and increase the likelihood of human error".

Currently there has been very little research done in the maritime industry to reveal the operator's real time capabilities and limitations resulting from varying workload levels. Research in the airline, automotive and power industry has come much further. A recent example from the power industry by Holzinger et. al [5], illustrates how it is possible to reduce the complexity of user-interfaces for safety-critical power-plant control systems.

In maritime software applications, especially the DP system, it is of interest to find a basis of the different types of information that is important to present in a GUI and identify the information that is not utilized during operation. This gives a basis for optimizing the amount of information presented to the user. The investigation of the foci areas of attention can be utilized to place important information where the operator intuitively rests the eye during operation. This will possibly reduce the overall workload on the operator as the search for information will be simplified.

\subsection{SIGNIFICANCE OF STUDY}

The principal reason for investigating foci of attention during safety critical operations is to map out the location of the areas of focus, how much time they spend in them and to investigate why they spend time in the specific areas. In addition, it would be interesting to investigate if they spend enough time in the correct areas.

If not, why not and how can we change the pattern of attention? This can lead to more efficient simulator training of crew and knowledge that can help towards improving the design of bridge equipment and bridge layouts. That will increase the safety for aft deck workers and also reduce stress for operators.

\section{BACKGROUND AND RELATED RESEARCH}

The role of the operator is mainly influenced by the design of the system: the automation in the system and its level of autonomy determines the work that is required from the operator. An operator monitors the process continuously during operations. The system is too complex to have a complete overview of what is happening in detail, so it is not possible for the operator to be aware of the exact status of all (sub-) systems at all times. This can cause lack of situation awareness once a fault occurs [6]. The operator will have to search through the system to find relevant details, which provide input for his/her understanding of the fault and its root cause. This search process consumes valuable time in the problem solving process.

During DP operations, one of the primary ways a DP operator (DPO) gathers information is by visually scanning DP control screens and the surrounding environment. Willems et al. [7] says;

"Visual scanning refers to a systematic and continuous effort to acquire all necessary visual information in order to build and maintain a complete awareness of activities and situations, which may affect the controller's area of responsibility".

Generally visual scanning consists of two types of eye movements; fixations and saccades. Fixations are the moments when the eyes are relatively stationary, taking in or "encoding" information [8]. Saccades are quick eye movements occurring between fixations [8]. When the fixations and saccades are combined it produces a scan pattern, which is called a scan path [9]. In addition to the above mentioned, it is also possible to measure eye blinks that gives interesting possibilities. Eye blinks are related to physiological factors including mood state and task demands and reflects the viewer's attention and tension [10]. Eye blink rate is inversely proportional to workload and high blink rate may indicate fatigue [8].

As measuring blink rate can be connected to physiological factors, the study of pupillary response can also reflect the operators' cognitive workloads. An increase in pupil size has been shown to correlate with increasing processing demands [11]. Supporting this, Szulewski et. al [12] states that the usage of eye-tracking (in this case Tobii eye-tracking glasses) can be valid to use in terms of measuring the percentage change of pupillary response to determine increased cognitive processing.

\section{EXPERIMENTAL DESIGN AND METHODS}

Little is known about where the operator focuses during DP operations. Due to the dynamic and relatively unpredictable nature of the real working environments, it is difficult to conduct controlled studies "in the wild". As such, we investigated operator's cognitive response to 
various tasks by simulating the tasks in a controlled environment (simulator). In this paper, we report our studies that collected and documented the foci areas of attention during simulated DP operations. While this has given us insights, we plan to validate these results in a real life environment during real DP operations (as much as possible given the safety-critical nature of the environment).

\subsection{METHODS}

In order to locate the areas of foci, we used both objective and subjective methods. The objective methods selected are:

- Study of ocular behaviour (eye-tracking)

- Study of pupillary response

Using eye-tracking gives advantages over other equipment as the technical readiness level is higher than that of other sensors. The subjective method chosen was to interview the operators post-experiment to gain insights into the reason why they focus in specific areas.

\subsection{METRICS}

The metrics that will be investigated are fixations, saccadic movements, pupillary response and eye-blink rate. Which will be further explained below.

\section{2 (a) Fixation}

Statistical analysis of fixation duration, fixation frequency, fixation duration max and standard deviation of the fixation duration can be done. These statistics are then used to observe if a correlation exists between the eye tracking metrics and the independent test variables.

\section{2 (b) Saccadic Movements}

Saccadic length can be used as an indirect workload measuring tool. Saccade length will decrease with increasing workload.

\section{2 (c) Pupillary Response}

Pupillary response can be an index of the amount of cognitive processing. Because increased processing load evokes greater pupillary dilation response. The problem in using this metric is it requires implementing several experimental controls such as keeping constant illumination of the experimental setting and the brightness of the stimuli.

\section{2 (d) Eye Blink Rate}

Eye blink rate is a frequency and duration of eye blinks are inversely correlated to mental workload. Higher blink rate may indicate fatigue. This metric also has the same problem as the pupillary response. For example influence of ambient light levels.

\section{2 (e) Scanpath}

Theoretically as workload increases the observed scanpath becomes less random.

\subsection{STUDY DESIGN}

The layout of the study will be as described below in three steps:

\section{Step 1 - formal details and training}

Before the experiment commenced the DPO was asked to sign a consent document. $\mathrm{He} / \mathrm{she}$ was given some basic training, a briefing of what to do according to the experiment protocol and a chance to familiarize with the equipment. The location for this was divided between the instructor station and inside the simulator.

\section{Step 2- experiment}

The DPO entered the room of the offshore bridge simulator where the simulator has a realistic aft bridge setup (Figure 2). This simulator is built around the previous version of Rolls-Royce bridge consoles. On the aft bridge there is a DP operator station where the operator was seated when the test commenced.

- The DPO started the experiment by taking command of the vessel.

- (S)He then entered DP mode and started the DP operation from a distance of 100 meters from the rig.

- When this is settled, the vessel can approach the rig.

- The DPO was then asked to stabilise the vessel at an appropriate distance from the rig and start loading/offloading one container. The amount of cargo was limited to due to time constraints.

- When finished loading/offloading, the operator departed from the platform and returned to a distance of $100 \mathrm{~m}$ from the rig.

- The operation ended when the vessel reached the distance of $100 \mathrm{~m}$.
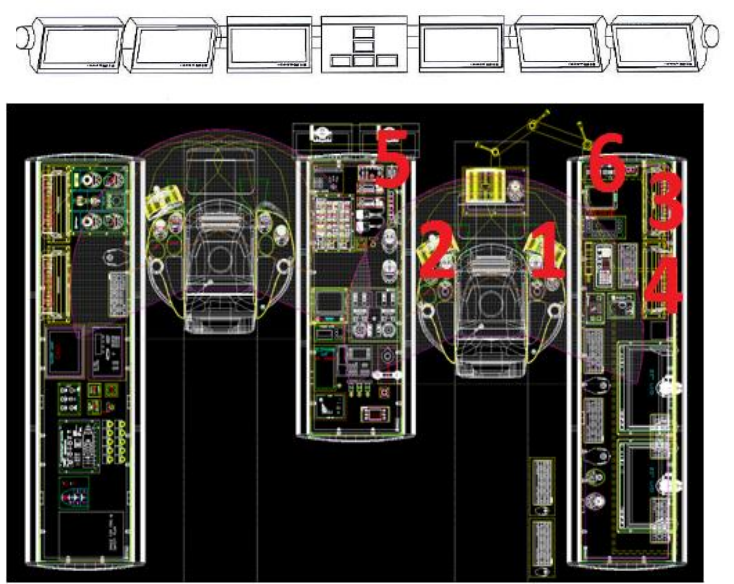

Figure 2: Aft bridge setup with six overhead VDUs and operational VDUs numbered from 1 to 6 .

Step 3 - evaluation 
The operator was, in cases where the reason behind foci of attention was unclear, interviewed to clarify according to the findings.

\subsection{HYPOTHESES}

With the above scenario in mind the areas of interest (AOI) investigated were categorized in two different categories:

- Outside Environment/Scenery

- Bridge equipment (controllers and monitors)

The experimental variables will be as follows:

\begin{tabular}{|l|l|}
\hline Independent variable & Dependent variable \\
\hline User- Experience & Foci of attention \\
\hline
\end{tabular}

Figure 3: Table describing variables

With the above information in mind (Figure 3), three hypotheses can be outlined:

1. The DPO's attention will mainly be on the aft deck of the vessel and the outside scenery during the operation. Expert operators will fixate more on the outside environment than novice operators.

2. When the DPO's attention is on the bridge equipment, it will be mainly on VDUs during the operation.

3. The DPO's pupillary response will increase when reaching a critical phase of the operation.

In addition it is also desirable to investigate the division of attention between the different scenery categories, scenery, off scenery and bridge equipment.

\subsection{TASKS}

There was a single set of tasks, described in section 4, carried out for all participants. We conducted a 2 x 1 design (Figure 4) where the focus will be to investigate foci areas of attention in concerning DP operations.

\begin{tabular}{|l|l|}
\hline Novice (4 participants) & DP operation \\
\hline Expert (4 participants) & DP operation \\
\hline
\end{tabular}

Figure 4: 2 x 1 study design

The participants' age, gender, experience and official title/education were registered in the consent.

\section{EXPERIMENTAL SETUP AND FINDINGS}

The study was carried out over a timeframe of five weeks. The subjects were all male, where four were experienced DP operators having their daily work life at sea and four subjects were novice, but did have a nautical study background and some hours of DP simulator training (Figure 5).

\begin{tabular}{|l|l|}
\hline Participant & Level of experience \\
\hline 1 & Expert \\
\hline 2 & Novice \\
\hline 3 & Expert \\
\hline 4 & Novice \\
\hline 5 & Novice \\
\hline 6 & Novice \\
\hline 7 & Expert \\
\hline 8 & Expert \\
\hline
\end{tabular}

Figure 5: Overview of participants in correct order with level of experience

The operation took approximately 30 minutes per participant. This excluded calibration and mounting of eye-tracking glasses, in addition to sketching out the scenario to the participant. The participant took place in the DP operator chair in the simulator (Figure 2), while the crane operator took place in the control room. The crane operator and the DP operator communicated via radio as done in real life situations [13].

The VDUs have been numbered from 1 to 6 and figure 2 illustrates the location of each VDU. A selection of different systems relevant to the operation are running on the VDUs as follows:

- VDU 1: DP remote control

- VDU 2: Propulsion control

- VDU 3: DP application

- VDU 4: DP application

- VDU 5: Showing distance to rig

- VDU 6: DP Joystick control

VDU 3 and 4 are running the same application, however VDU 3 are partly occluded by VDU 1 . This leads, we anticipated, to VDU 4 being more frequently used for monitoring the system than VDU 3. It is also important to notice that all VDUs have touch screen functionality. 


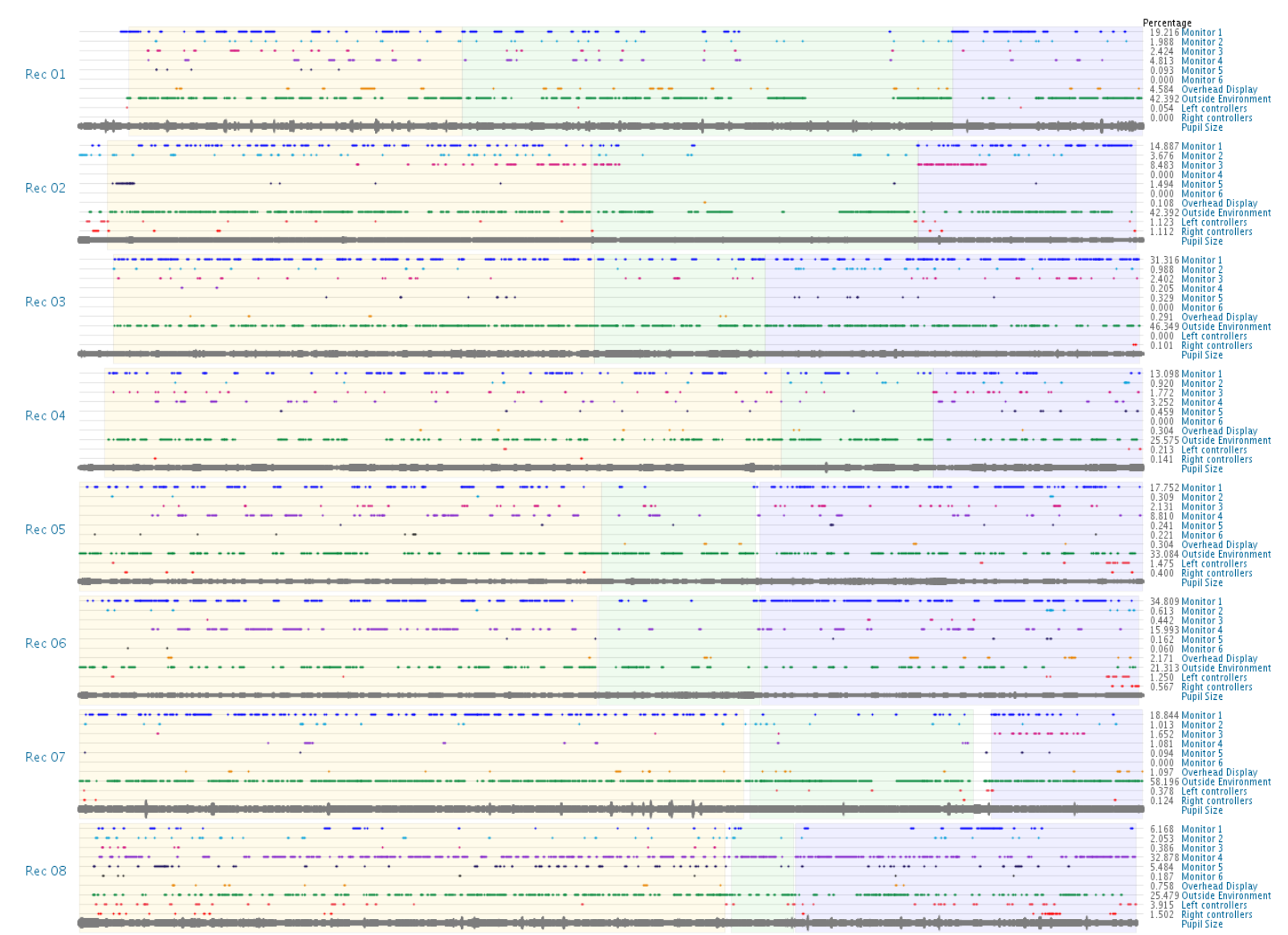

Figure 6: Visualization of collected eye-tracking data

\subsection{FINDINGS}

\section{1 (a) Interpretation of the Visualisation Map}

The visualisation map (Figure 6) is divided into eight different rows representing the different recordings/participants and three columns representing approaching the oil rig (yellow), positioning the vessel (green) and retreat from the oil rig after completed operation (blue).

\section{Outside Environment}

When looking at the visualisation of all collected eyetracking data per participant, one can clearly see that the expert participants ( $\operatorname{Rec} 01,07 \& 08)$ spend more time looking out of the windows than the novice participants, as anticipated. The green line in the visualisation map illustrates time spent looking at the outside environment (aft deck and scenery). In addition VDU 1 (blue line) and 4 (purple) catches the operator's eyes most frequently. The tasks carried out on VDU 1, the DP system remote control, are directly connected to controlling the vessel. VDU 4 holds a 3D visualisation of the vessel with set point, distance and position reference information. This VDU is mainly used for monitoring, as it is too far away for direct interaction.

\section{Levers}

The operator interacts with the levers (left and right levers indicated with red dots) continuously throughout the operation. However they seldom glance down on the levers or the levers' buttons. The visualisation shows quite clearly that the majority of the operators spend less than $1 \%$ of their time glancing down at the levers. If the vessel drifts off its set point or any other event occurs, an alarm will sound triggered from the software application and the lever. The operator must then attend to both locations to reset the alarm. This requires the operator to look down at the lever to push the relevant buttons. Tactile feedback and experience can have an impact on the need for looking down at the lever. When the operator is familiar with the lever and the location of the particular buttons, their visual attention is not required to be able to orientate and silence e.g. alarms.

\section{Visual Display Units}

During the different stages of the operation, the operators use the VDUs differently. The visualisation map illustrates that during the approach to the oil rig, the VDUs are frequently used (especially VDU 1 and 4 as indicated earlier). When the operator has positioned the vessel next to the oil rig the operators glances briefly at the displays to be reassured that the vessel is still in position. The visualisation map indicates that novices check the VDUs more extensively, also during loading and offloading of cargo when the vessel is in position. When the operation is over and the vessel backs away from the oil rig, the frequency of looking at the VDUs increases, especially when looking at VDU 1.

\section{Pupil Dilation}

It was also possible to collect an indication of pupil dilation during the operation. Pupil dilation can give an indication of stress and cognitive load on the operator. 
From the visualisation (pupil dilation indicated with a thick grey line) we can in this case see that it is the experts that have a more varying pattern of pupil dilation showing more peaks. The reason behind this is still unclear and will be investigated in more detailed at a later stage.

A more detailed analysis of the above data will be described below.

\section{1 (b) Statistical Analysis}

The average of fixations of total recording time, fixation duration across the different stages of the operation, average dwell per focal area, percentage of fixation per focal area and total time fixating on focal areas per operational stage were measured. With this information it was possible to map out the focal areas and difference between experts and novices during the operation.

The percentage of fixations were measured according to recording time. Since a part of the overall recording time are spent not dwelling at any particular focal area or object at all, the percentage does not add up to become $100 \%$.

\section{Total Time of Fixation}

When comparing the total time fixating per area, it becomes clear that less than $3 \%$ of the operators' fixation time was spent looking at the levers (left and right controllers). See Figure 7. The same trend shows for VDU $2(0.75 \%), 3(2 \%), 5(1 \%), 6(0 \%)$ and the overhead VDUs (1.5\%). This indicates that the information displayed in these VDUs are not very important to the operator during the DP operation.

The remaining time was spent fixating on VDU 1, 4 and the outside environment (scenery and aft deck). In total $18.5 \%$ of the operators' time was spent monitoring and interacting with VDU1 (DP remote control) and 9\% monitoring the information displayed on VDU 4 (DP 3D scene). Concerning the outside environment it clearly was the area of interest that, as anticipated generated the highest amount of fixations, where the operators spent in average $35 \%$ of their time looking out of the windows.

When comparing total times of experts versus novices in the different areas of interest, the same trend as described above was reflected. While there is wide variation, on average it appears that experts spent more time looking at the outside environment than the novices (Figure 8 ). The heat map in figure 10 illustrates fixations of one of the expert participants. The red colour indicates longer fixations.

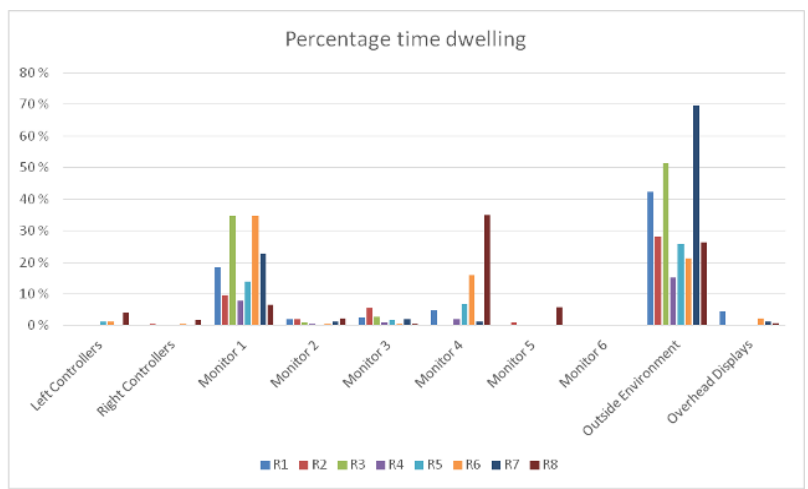

Figure 7: Total time comparison of fixations throughout the operation.

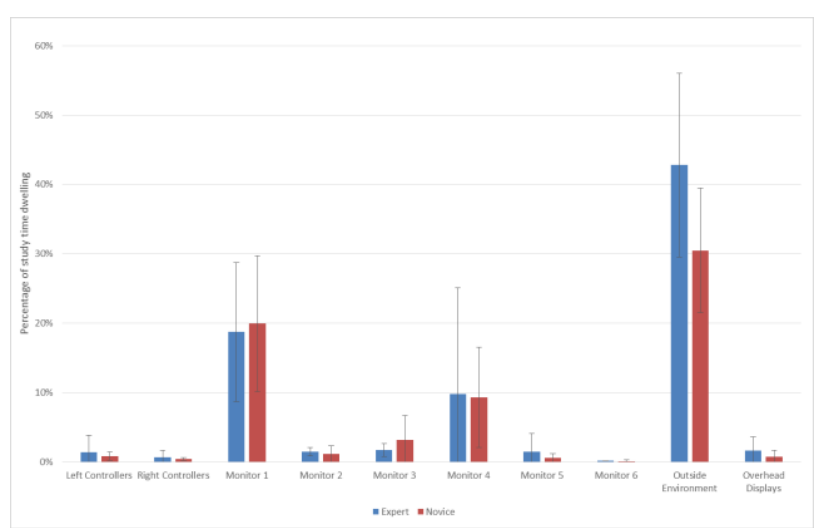

Figure 8: Percentage of study time fixating on each focal area divided between experts versus novices (blue = expert, red $=$ novice, $95 \%$ confidence level).

\section{Average Time of Fixation}

When looking at the average time of fixation, there are clear differences between novices and experts. The data shows that novices fixates less on equipment and outside environment than experts. None of the calulations on average fixation time show any significant results, but give a clear indication that experts focuses more on the more important equipment and areas than novices.

When breaking this down into focusing on the areas of intrest that has emerged (VDU 1, 4 and the outside environment) we can report (Figure 9) that :

- Mean total length of fixation for experts on VDU 1 $(\mathrm{n}=4)$ averaged $3238 \mathrm{~ms}(\mathrm{~s}=1198 \mathrm{~ms})$, while for novices $(n=4)$ averaged $2260 \mathrm{~ms}(\mathrm{~s}=1882 \mathrm{~ms})$.

- Mean total length of fixation for experts on VDU 4 $(\mathrm{n}=4)$ averaged $2270(\mathrm{~s}=688 \mathrm{~ms})$, while for novices $1863 \mathrm{~ms}$ ( $\mathrm{s}=1270 \mathrm{~ms})$.

- Mean total length of fixation for experts on the outside environment $(\mathrm{n}=4)$ averaged 3875 ( $\mathrm{s}=$ $1662 \mathrm{~ms}$ ), while for novices $2378 \mathrm{~ms}$ ( $\mathrm{s}=742 \mathrm{~ms}$ ).

Showing that expert operators tended to maintain their individual glances for longer both on the principle VDUs and on the outside environment. See figure 10. 


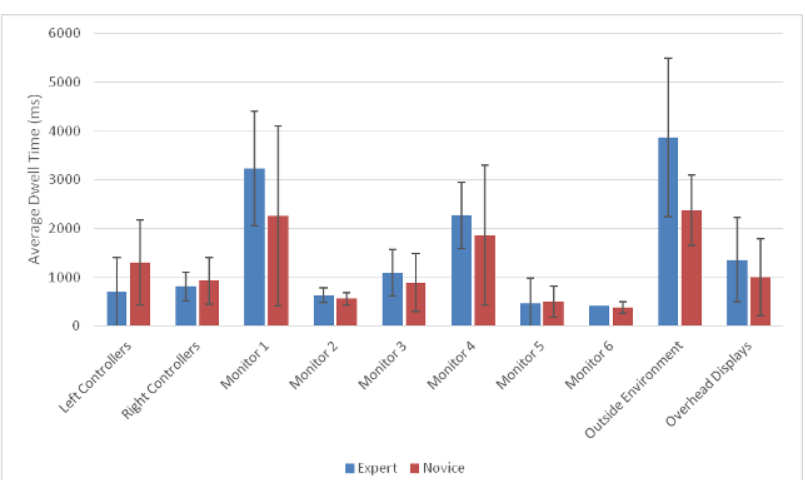

Figure 9: Average fixation times per focal area with $95 \%$ confidence level.

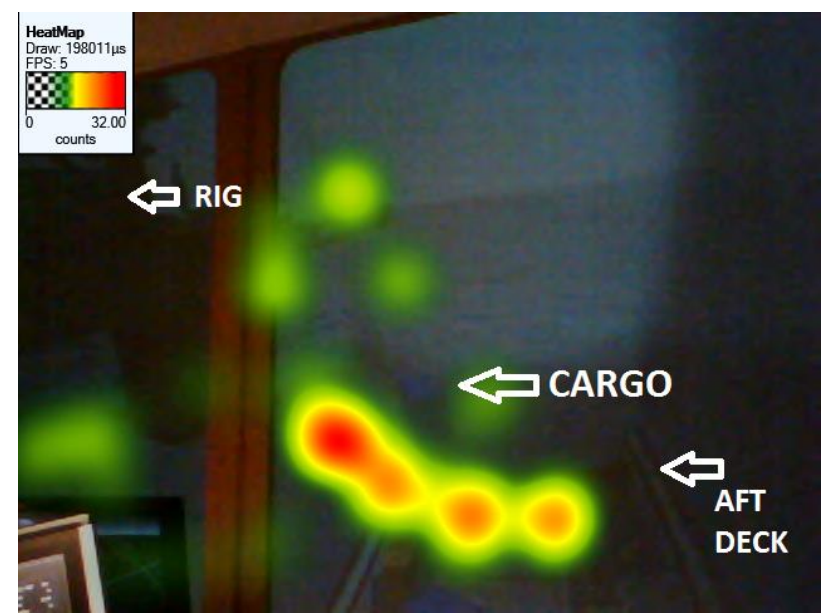

Figure 10: Heat map showing fixations in the outside environment by an expert participant.

\section{Total Time of Fixation per Operational Stage}

The DP operation consists of three different stages, as mentioned above (approaching the oil rig, positioning the vessel and retract from the oil rig). When studying the three different operational stages, different fixation patterns appear from the data collected.

The visualisation map gave an indication of this, however when dividing the total time of fixation on the focal areas into the different operational stages (see Figure 11), the three main focal areas stand out clearly, which is as indicated earlier, VDU 1, 4 and the outside environment. It is also possible to see that the amount of fixations in all main focal areas are at a much higher level during the actual approach towards the oil rig. The moment the vessel has been positioned, the activity decreases and the main operation is now happening on the aft deck where deckhands prepared cargo for loading/offloading. The operator's main task is to monitor the activities and make sure that the vessel does not drift off. When the cargo handling has been completed the activity pick up and the amount of fixations then increases, however not to the same level as when approaching. This falls natural, as the retract is far less safety critical than the approach.

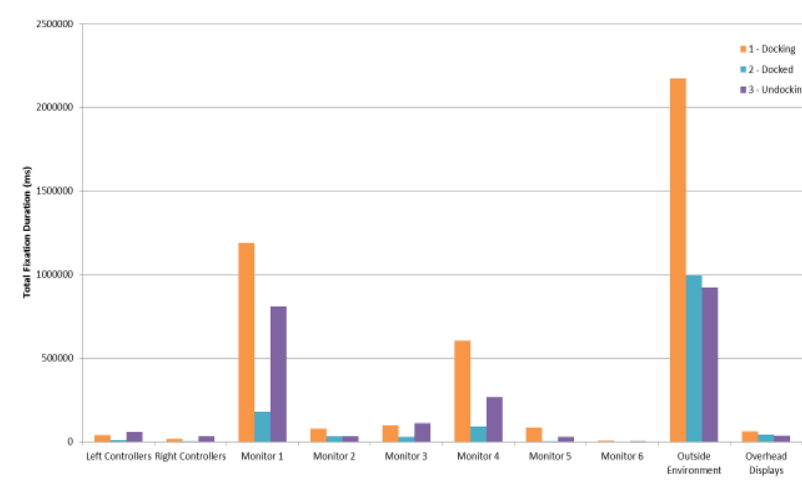

Figure 11: Total time of fixation on focal areas per operational stage $($ approach $=$ orange, in position $=$ blue, rertact $=$ purple)

\section{CONCLUSIONS}

When looking at the collected data and the results that emerged, there are clear indications that expert operators do spend more of their total time during the operation, fixating on the outside environment and important equipment. Novices fixate less, which reflect their level of experience as they do not have the basis of knowledge that expert operators have on what to look for and possible dangers to be aware of.

Our main goal for this experiment was to investigate which focal areas the operators focused on during a standard DP operation. We found that the outside environment (scenery and aft deck), as anticipated, was the main focal area. The expert operators focused longer and more frequent on this focal area than the novices. This confirms our first hypothesis, that the DPO's attention will mainly be on the aft deck and on the outside scenery during the operation. It also confirmed that expert operators fixated more outside than the novice operators.

The additional focal areas were, also as anticipated, on the VDUs. VDU 1 and 4 gave the highest amount of fixations which confirm our second hypothesis that when the operators' attention are on the bridge equipment, it will mainly be on the VDUs. With the current bridge setup, VDU 1 and 4 are important equipment in use during the DP operation. When analysing the recordings, it seemed like the operators preferred to glance at VDU 4 instead of VDU 3, as it was closer and easier to read (and not occluded by VDU 1). In addition, the operators also interacted with the VDU. This emphasizes the importance of having the interaction surfaces in close proximity to the user, both for increased readability and usability. In these areas there are apparent possibilities of improvement in terms of arranging a new bridge setup, which will be discussed in the next section.

Our third hypothesis concerns pupillary response. From the data collected one could see variations in pupil dilation, however the amount of data was complex and fluctuating. From the visualisation map it was possible to 
extract that expert operators experience more variation in pupil dilation than novice operators. This can correlate with the fact that experts actually have a larger amount of fixations all together. Even though we see indications that the DPO's pupillary response increases when reaching a critical phase of the operation, we cannot confirm this, as it demands closer investigation.

\section{SUGGESTION OF IMPROVEMENTS}

The above results introduce possible improvements from the current aft bridge setup (figure 2) to the new aft bridge setup (figure 12).

The two small VDUs (VDU 1 and 2) that, on today's setup, are fitted on the armrests of the operator's chair, cause occlusion of the aft deck and of other VDUs. By removing the armrests all together and moving all controllers (levers, button panels and VDUs) closer to the user, it is possible to reduce the amount of necessary VDUs and open up the FOV to the aft deck.

VDU 2 that has a more in-frequent interaction pattern than VDU 1, can be moved to the 10" VDU to the right of the operator, while the main interaction surfaces get an increased size. The DP remote control (VDU 1) becomes obsolete as the operator can both operate and monitor the main DP application interface directly, without having to interact with two VDUs (VDU 1 and 4) and turn to the right for monitoring the system.

By introducing these changes to the setup, it is possible to combine the two main bridge environment focal areas (VDU 1 and 4), into one focal area. The operator can then relate to the outside environment and one interaction surface in the bridge environment during the DP operation, that are right in front of the operator. This reduces visual scanning of the aft bridge environment and less areas to maintain situational awareness of. This can have an impact on the operators workload during operation and critical phases.

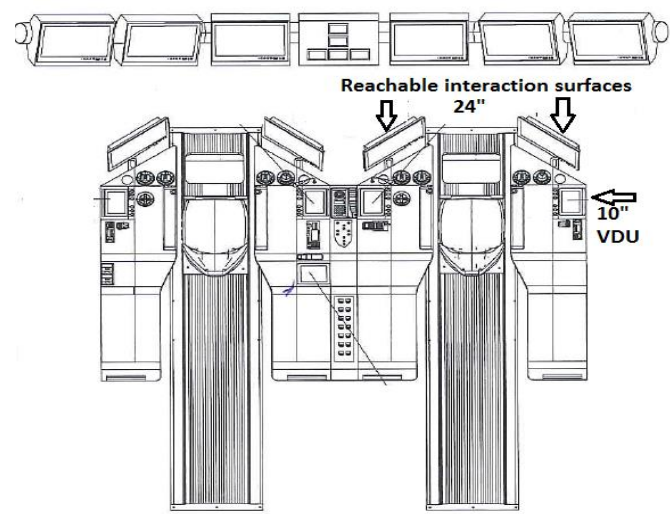

Figure 12: Rolls-Royce Unified Bridge layout with improvements implemented.

\section{FUTURE WORK}

The next step in this research will be to redo the tests introducing environmental effects such as current and wind, to compare if the visual patterns change noticeably for a standard operation. In addition it is desirable to redo the experiment using a new aft bridge setup to investigate if the scan-patterns become less frequent. When this has been documented, the investigation will go further into detail on the graphical user interfaces of the DP application.

\section{ACKNOWLEDGEMENTS}

The authors would like to thank the Norwegian Research Council for funding this research, our research assistant Qin Liang from the Maritime Human Factors Laboratory for helping with collection of data and Arnt Håkon Barmen and Tron Resnes, responsible for training of maritime crew at Aalesund University College, for gathering relevant participants.

\section{REFERENCES}

1. BJØRNESETH, F.B., et al., ' Towards an experimental design framework for evaluation of dynamic workload and situational awareness in safety critical maritime settings', Proceeding BCS-HCI'12 Proceedings of the $26^{\text {th }}$ Annual BCS Interaction Specialist Group Conference on People and Computers, pages $309-314$.

2. BRAY, D. ' Dynamic Positioning $-2^{\text {nd }}$ Edition, OilField Publications Ltd, London, UK.

3. IMCA, 'Station Keeping Incidents reported for 2006.', International Marine Contractors Assocoation.

4. NOCERA, D. et al., 'A Random Glance at the Flight Deck: Pilots' Scanning Strategies and the Real-Time Assessment of Mental Workload', Journal of Cognitive Engineering and Decision Making, Vol.1, p 271 -285, 2007.

5. HOLZINGER,A., et al., 'On complexity reduction of user interfaces for safety-critical systems' International Conference on Availability, Security, and Reliability, IFIP Cross Domain Conference, LNCS 7465 (2012), 108-122

6. TJALLEMA, A., VAN DER NAT, C., 'The Road to Eliminating Operator Related Dynamic Positioning Incidents', Dynamic positioning conference, Vol. 2., 2007

7. WILLEMS, B., et al., 'Air Traffic Control Specialist Visual Scanning II: Task Load, Visual Noise, and Intrusions Into Controlled Airspace', Technical Note (DOT/FAA/CTTN99/23), FAA, 1999. 
8. POOLE, A., BALL, L.J. 'Eye Tracking in Human-Computer Interaction and Usability Research: Current Status and Future Prospects.' Chapter in C. Chaoui(Ed.): Encyclopedia of HCI. Pennsylvania: Idea Group, Inc., 2005.

9. ELLIS, K., 'Eye Tracking Metrics for Workload Estimation in flight Deck Operation', Thesis, University of Iowa, 2009.

10. BRUNEAU, D., et al., 'The eyes never lie: The use of eye tracking data in HCI research', $\mathrm{CHI}$ 2002, Workshop on physiological computing, Minnesota, USA, 20-25 th of April, ACM Press, 2002.

11. BEATTY, J., 'Task-evoked pupillary responses, processing load, and structure of processing resources' Psychological Bulletin, 91 , pp. 276292, 1982

12. SZULEWSKI, A. et al., 'Increasing Pupil Size is Associated with Increasing Cognitive Processing Demands: A Pilot Study Using the Tobii Glasses Eye Tracker', Poster Presentation, Eye-Tracking Behaviour, 2012.

13. BJØRNESETH, F.B., 'Assessing the Effectiveness of Direct Gesture Interaction for a Safety Critical Maritime Application', $P h D$ thesis, University of Strathclyde, Glasgow, UK, 2010.

\section{AUTHORS BIOGRAPHY}

Dr Frøy Birte Bjørneseth holds the current position of maritime HF and HMI specialist at Rolls-Royce Marine AS in Norway. She is responsible for ensuring and maintaining good usability and human factors of current and future Rolls-Royce products, both physical equipment (bridge consoles, operator chairs and levers) and software interfaces.

Loraine Clarke is currently studying for a $\mathrm{PhD}$ in Computer Science at University of Strathclyde in Glasgow. Her research is into multimodal interaction in the context of museums interactive exhibits. As such, she had developed considerable experience and skills in video analysis and was responsible for analysing the eyetracking data in this experiment.

Dr Mark David Dunlop is a senior lecturer at University of Strathclyde in Glasgow. He leads the Mobiquitous lab research group into usability of mobile and ubiquitous computing. His main areas are research within mobile usability, particularly smartphone usability, and novel input methods.

Dr Sashidharan Komandur holds a position as associate professor at Aalesund University College and leads the Maritime Human Factors Laboratory. His main area is within maritime research. 